Slavica

bruxellensia

\section{Slavica bruxellensia}

Revue polyphonique de littérature, culture et histoire

slaves

$11 \mid 2015$

Littérature et philosophie

Vers une stylistique de l'acte. La querelle de Karol Irzykowski et Stanisław Brzozowski à propos du Trésor de Leopold Staff dans le contexte des philosophies polonaise et allemande

\title{
Michał Mrugalski
}

Traducteur : Katia Vandenborre

\section{(2)enEdition}

Journals

Édition électronique

URL : http://journals.openedition.org/slavica/1715

DOI : 10.4000/slavica. 1715

ISSN : 2034-6395

Éditeur

Université libre de Bruxelles - ULB

Référence électronique

Michał Mrugalski, « Vers une stylistique de l'acte. La querelle de Karol Irzykowski et Stanisław

Brzozowski à propos du Trésor de Leopold Staff dans le contexte des philosophies polonaise et

allemande », Slavica bruxellensia [En ligne], 11 | 2015, mis en ligne le 15 février 2015, consulté le 30 avril 2019. URL : http://journals.openedition.org/slavica/1715 ; DOI : 10.4000/slavica.1715

Ce document a été généré automatiquement le 30 avril 2019.

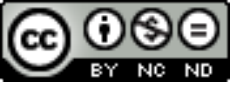

Les contenus de Slavica bruxellensia sont mis à disposition selon les termes de la Licence Creative Commons Attribution - Pas d'Utilisation Commerciale - Pas de Modification 3.0 France. 


\title{
Vers une stylistique de l'acte. La querelle de Karol Irzykowski et Stanisław Brzozowski à propos du Trésor de Leopold Staff dans le contexte des philosophies polonaise et allemande
}

\author{
Michał Mrugalski
}

Traduction : Katia Vandenborre

Il existe un moment, un moment où le chantre doit s'arrêter dans son développement ou devenir un poète-prophète, où se termine l'agencement du drame et commence la tragédie. Une tragédie dont on n'est plus l'auteur, mais le héros et la victime.

Stanisław Brzozowski ${ }^{1}$

\section{La Théorie de la tragédie : la relation de la théorie et de la pratique}

Lepold Staff a écrit Skarb. Tragedia w trzech aktach (Le Trésor. Tragédie en trois actes) en $1904^{2}$. Par opposition aux écrits de Karol Irzykowski, qui évoque souvent Le Trésor de Staff, cette pièce ne joue pas un grand rôle dans l'œuvre imposante, inquiétante et intimidante de Stanisław Brzozowski. Celui-ci a consacré à ce drame une petite recension pathétique ainsi que quelques mots cinglants qui cernent les caractéristiques de Staff, faisant partie du livre de Brzozowski de 1907 Kultura $i$ życie (La Culture et la vie) ${ }^{3}$. Le sous-titre du drame, "tragédie en trois actes", a éveillé l'indignation de Brzozowski. À chaque fois qu'il évoque le Trésor, Brzozowski reproche au poète le caractère non tragique de sa 
prétendue «tragédie ». Le Trésor ne remplit pas les conditions de base du tragique que Brzozowski connaît et qu'il puise - comme il le prétend lui-même - de Friedrich Nietzsche, bien que l'analyse de l'argumentation montre plutôt la copaternité de Georg Wilhelm Friedrich Hegel ${ }^{4}$. La formulation des règles et principes de la tragédie occupe la plus grande part de la recension de Brzozowski ainsi que la fin de la caractérisation de Staff. La philosophie du tragique était un asile dans lequel était conservée la poétique normative. Cela était lié au poids de la philosophie de la tragédie, tant pour les grands systèmes philosophiques, par exemple le système de Hegel, que pour les penseurs antisystèmes, comme par exemple Kierkegaard ou Nietzsche. La théorie de la tragédie a toujours été la théorie de la relation entre théorie et pratique. Elle modelait le passage de l'intention à l'action ou la confrontation d'une reconnaissance théorique avec la factualité . La théorie de la tragédie chez Brzozowski est similaire: la théorie de la tragédie est une théorie du rapport entre la vision du poète et sa vie. Ajoutons : une vie remplie de travail. Chez Brzozowski, le concept du «tragique » se définit par rapport aux concepts de «liberté », de "travail », de "vie », et "d'acte " « fatum » ni à «l'Être ». Au nom de cette nouvelle liberté, il faut construire cette tragédie qui est justement le dépassement du fatalisme. La tragédie est un jugement de la vie en général sur la vie humaine individuelle qui mesure son intensité. Elle est «une intensification de la vie jusqu'à l'autodestruction orgiaque à travers l'auto-enivrement $»^{7}$. La "tragédie » de Staff est une usurpation. Elle n'atteint même pas la vie, puisque tout Staff peut être décrit dans les termes d'un idéalisme déraillé : tout Staff est comme une parodie du «moi » fichtien ${ }^{8}$ qui, au lieu de faire du monde la matière de son devoir, apparait soudainement incapable d'établir son être dans l'acte primaire de Tathandlung, d'accomplir un « acte » quand le moi annonce au moi la formule Grundsatz « le moi devrait être ». Le rêveur cependant, tout comme le Staff pseudo-tragique par exemple, a peur de l'être. Pour réaliser l'impératif catégorique de l'acte das Ich soll sein («le moi devrait être »), une " belle âme » devrait choisir un des « êtres » possibles et être seulement ellemême, agissant. Elle préfère donc perdre la vie plutôt que toutes ces inépuisables possibilités avec lesquelles joue cet éternel spectateur et metteur en scène, possibilités qui jouent devant et pour lui. Dans la vie, dans le meilleur des cas, il reçoit le rôle d'un étrange inconnu venu du futur', quand, selon Brzozowski, le travail devient enfin ce qu'il est dans son essence profonde et affirmée : un synonyme de liberté. En attendant, dans le monde du travail réifié, non pas le rêveur, mais seul "l'homme réel, guerrier et vagabond " a le droit et la possibilité d'écrire des tragédies. Dans Le Trésor de Staff, il manque même "l'effort spirituel, l'héroïsme intérieur, le tragique de l'âme $»^{10}$. Plutôt qu'une nécessité concrète - et "la nécessité dans l'art est le dévoilement que le vécu créatif s'enracine dans la vie même» - nous recevons un schéma et une abstraction: "l'action du Prométhée d'Eschyle, d'Edipe de Sophocle, de Macbeth de Shakespeare (...) est définie nulle part et partout, (...) mais nous ne pouvons localiser Le Trésor de Staff en dehors de la tête de l'auteur $»^{11}$ :

(...) rien de plus illusoire que les personnages du Trésor de Staff: ce Guide, ce Gardien inébranlable, cette Étrange, ce sont de simples jeux d'impuissance. Ceci n'a rien à voir avec l'art de créer des forces et des hommes vifs et plus vifs que la vie, tout comme la naissance du Trésor n'a rien à voir avec la création du tragique. Celleci naît des réalisations les plus hautes, ne fût-ce que de la vie intérieure. Tandis que Le Trésor est né des réflexions sur les possibilités vitales, des pensées sur ce que peut être l'idéal, pas un idéal précis et singulier, mais l'idéal en général. Même compris ainsi, cet idéal pourrait devenir le bourgeon du tragique, mais sa conception devrait être vécue. (...) Les adhérents à l'idéal, les gardiens du "Trésor", le décrivent 
comme « Inconnu ». Ils ont suivi les cours du néokantisme, c'est sûr, mais ils n'ont

pas eu affaire au tragique, à la tragédie ou à la révélation visionnaire de la vie. ${ }^{12}$

La colère contre le manque de vie s'est consumé sur quelques pages et Brzozowski n'est plus jamais retourné au Trésor.

2 «Brzozowski, s'il avait regardé avec plus d'attention la symbolique du Trésor, écrit Irzykowski, aurait retrouvé en elle beaucoup de ses conceptions » ${ }^{13}$. Et plus loin : "J'avais moi aussi quelques similarités fortuites avec Le Trésor de Staff. Mon œuvre Sny Marii Dunin (Les rêves de Maria Dunin) comporte la même extériorisation symbolique du jeu de la conscience : La Fratrie de la Grande Cloche. Mais chez moi l'affaire est plus satirique et contient plusieurs fonds. " ${ }^{14}$ Irzykowski mentionne souvent Le Trésor de Staff et entre presque toujours en polémique avec Brzozowski, comme si le nominaliste et le disciple des empiriocritiques voulait créer chez le lecteur le réflexe d'associer le nom de «Brzozowski » au Trésor. Il construit un complexe d'associations : Le Trésor-BrzozowskiIrzykowski-l'acte-le travail-le programme-le but-l'abstraction-le concret. Son jugement sur Le Trésor change - de la critique cinglante dans le livre sur Friedrich Hebbel à sa présentation comme modèle du drame allégorique dans la recension "OkrĘt sprawiedliwych »(«Le Navire des Justes ») de Mikołaj Jewreinow ${ }^{15}$. Partout où Irzykowski mentionne Le Trésor apparaît directement Brzozowski : c'est une constante.

\section{Le triomphe et la défaite de l'allégorie}

Il ne convient pas de juger méchamment le fait que, pour Brzozowski, Le Trésor constituait à peine un prétexte pour exprimer ses propres idées théoriques sur l'essence de la tragédie, de l'acte et du vécu. Dans les deux textes consacrés au drame, Brzozowski ne perd pas des yeux Le Trésor, même pour un moment, au point que la référence a un caractère négatif, voire le caractère du négatif photographique. Brzozowski dit ce que la tragédie de Staff n'est pas, et c'est la meilleure recension que Staff pouvait souhaiter. La qualification générique contenue dans le sous-titre "tragédie en trois actes » était en effet une provocation visible envers toute la philosophie du tragique et la critique littéraire de son temps. Staff contestait la poétique normative qui légitimait des secousses dionysiaques ou un savoir absolu autre sur l'élément avec lequel l'humanité se bat de manière tragique. Formulant à l'occasion du Trésor la seule définition juste du tragique en tenant en l'occurrence compte « des grands intérêts vitaux simplistes et synthétiques de l'humanité $»^{16}-$, Brzozowski a compilé de cette manière des fragments de Philosophie des Tragischen et de la Lebensphilosophie allemande, pour donner une antithèse à la thèse de Staff qui affirmait que «Le Trésor était une tragédie ». Ainsi les caractères formels du Trésor, pétris par Staff de ses concepts sur la tragédie étaient, grâce à Brzozowski, mis en relief et consolidés. On peut, à partir du négatif, faire ressurgir non seulement l'œuvre "sur le fond", mais aussi le geste stylistique du critique qui, comme le suggère Irzykowski, a fait tout ce qu'il pouvait pour ne pas y trouver ses propres conceptions.

Trois traits peu spécifiques lient Le Trésor de Staff avec la tragédie : la forme du drame; les dialogues versifiés, maintenus pendant toute la trame de l'histoire, mis à part dans l'intermezzo satirique, dans un registre élevé ; et enfin le fait de se diriger précisément vers la catastrophe. Cette «tragédie en trois actes » n'impose pas d'associations avec la tragédie grecque, que les philosophes allemands considéraient comme le modèle de la tragédie, reprochant au contemporain son manque de tragique. Le Trésor est quant à lui plus proche du Trauerspiel moderne, opposé par Walter Benjamin à la tragédie sensu graeco 
. Hugo von Hofmannsthal, proche de Staff, a renouvelé le Trauerspiel baroque. Benjamin cite à cet égard Der Tor und der Tod (Le But et la Mort, 1893) d'Hofmannsthal dans ses esquisses aux Thèses sur le concept de l'Histoire: « lire ce qui n'a jamais été écrit », voici une phrase d'un historien matérialiste ${ }^{17}$.

La différence entre le Trauerspiel et la tragédie peut être plus facilement saisie du point de vue de la relation entre le mot et l'acte. La langue de la tragédie grecque et le contexte spatiotemporel construit pour les discours des personnages était fait de sorte que chaque mot était le performatif le plus fortuné et en même temps fatal. Trauerspiel, littéralement le « jeu du deuil », exprimait cependant la tristesse infinie du discours incurablement vide, abstrait, se languissant du concret ${ }^{18}$. L'allégorie est une figure focalisant toutes les figures, et qui rend vivant le langage déchanté. Avant qu'elle n'acquière du sens grâce aux analyses, elle peut être définie comme l'association purement arbitraire d'un élément figuratif avec un élément signifiant, de sorte que l'arbitraire devient un symptôme caché (par exemple sous le couvert d'une connaissance ésotérique des connexions secrètes entre les images), disant tout sur la maladie de la langue. Bien que Benjamin et ses interprètes contemporains tiennent à occulter ce fait, la différence entre le discours complet de la tragédie et le discours ravagé du Trauerspiel repose en fait principalement sur le point de départ, sur la bonne vieille distinction formulée par Friedrich Wilhelm Joseph von Schelling, Johann Wolfgang von Goethe et Georg Wilhelm Friedrich Hegel entre un symbole motivé et une allégorie arbitraire ${ }^{19}$, distinction croisée avec le diagnostic que fait Schiller de l'époque sentimentale et désabusée.

À tous les niveaux de sa construction, Le Trésor est une allégorie. Comme l'a fait remarquer Brzozowski, les personnages et leurs discours sont allégoriques. L'espacetemps du monde représenté ( «Le temps et le lieu des événements est indifférent. Peutêtre "jamais et nulle part", peut-être "en nous chaque jour" " ${ }^{20}$ ) est allégorique, de même que l'action de la pièce. Celle-ci raconte comment un peuple menant, au nom d'un mystérieux trésor caché, des combats sans fin contre des barbares, décrits comme des personnifications des instincts, perd sa pureté et donc le droit de lutter au nom du trésor. Alors que le peuple veut vérifier ce que ce trésor est vraiment, le dernier Gardien et l'Étrange, inflexibles et désireux de garder le secret, empêchent le sacrilège en faisant tout exploser, y compris eux-mêmes, le trésor et l'ensemble du peuple. Typique du style allégorique, l'accumulation de tropes qui s'ajoutent les unes aux autres jusqu'à l'engorgement du sens commande de définir l'inconnu par l'inconnu, l'allégorie par d'autres allégories. Ainsi, le mystérieux trésor est comparé à un miroir dans lequel seul un homme vertueux peut se voir ou à un livre vierge sur les pages blanches desquelles seuls les actes les plus nobles peuvent être inscrits. En quoi le trésor consiste-t-il finalement ? Et s'il a existé, existe-t-il toujours ? Nous ne le saurons jamais, car le Gardien et l'Étrange ont emporté le trésor - certainement tout aussi impénétrable pour eux - dans la tombe, emmenant avec eux le peuple trop curieux. On sait que le trésor exige des victimes et que le peuple est fatigué de la grande responsabilité collective. Par conséquent, le conflit de base de la pièce se joue entre les exigences du trésor et le bonheur personnel. Autrement dit, à un niveau un peu plus abstrait, la pièce de Staff confronte l'éthique du droit avec l'éthique eudémonique. Brzozowski préconise l'éthique du devoir contre l'éthique eudémonique qu'il méprise avec autant d'éloquence que le Gardien et l'Étrange ${ }^{21}$. Chez Staff, ce conflit d'éthiques est transposé dans plusieurs conflits spécifiques : le confit entre une mort noble au nom d'idéaux et la préservation de 
la vie; entre la force de la foi et la force physique ; entre l'idéal et la corporalité ; entre l'abstraction et le phénomène ; enfin, entre le mystère et le concret.

7 Le Gardien dit : « À quoi bon / savoir ? Après tout, tout mal a un nom... $»^{22}$ il dirige cette question rhétorique non seulement à l'adresse des autres personnages de la pièce, mais aussi au spectateur, dans le mesure où celui-ci comprend que dans Le Trésor - comme l'a saisi Irzykowski - la «dissimulation» est une «forme » qui se constitue au moyen du contenu et de l'idée de l'œuvre ${ }^{23}$. Pour parler la langue du formalisme dans ses débuts ${ }^{24}$, la dissimulation constitue la dominante de la pièce : un élément du tout est mis en avant, de sorte que tous les éléments restants subissent une déformation en raison de l'accent qui est mis sur la dominante-dissimulation.

Dans l'intermède comique, la partie inaliénable du Tauerspiel baroque avec l'Hanswurst enragé ${ }^{25}$, la pièce de Staff devient ouvertement réflexive et tournée vers elle-même : les personnages de l'intermède savent qu'ils sont nés dans l'esprit de l'auteur en tant qu'allégories de ces obscures allégories qui prennent part à l'action principale de la pièce. Mais le conflit fondamental qui oppose les principaux camps du drame au nom du mystère, l'abstraction, la foi et de l'acorporalité est justement un conflit réflexif, un conflit de stylistiques : allégorique et réaliste, mais peut-être aussi allégorique et concrètement tragique, c'est-à-dire tel que l'exigeait Brzozowski, ancré dans la vie et le vécu.

Ce trésor stérile, figé, pétrifiée ${ }^{26}$;

est-ce que dans son impuissance repue, lourde à l'excès, dans ce ventre bouffi de contenu d'or,

dans cette tombe qui ne cesse de se déglutir elle-même

dans cet animal alourdi à la tête dorée

il y a ne serait-ce qu'une graine pour les bourgeons de la vie

et de l'air ne serait-ce que pour une bouffée dans ce vide ? ${ }^{27}$

9 Le trésor apparaît comme une anti-vie, une pure figure allégorique soulignant sa séparation de tout ce qui relève de la "vie ». La défense désespérée de l'éthique du devoir par les allégories du Gardien et de l'Étrange est le reflet du conflit des styles.

Le Trésor est né de réflexions non seulement sur l'idéal, mais aussi sur le style: un style allégorique élevé qui serait capable d'exprimer le mystère de l'idéal de tous les idéaux partiels (« de vie »). Car la vie est toujours intéressée, eudémonique, soucieuse du bienêtre. Et si Le Trésor de Staff contient en général des péripéties tragiques, une reconnaissance tragique et une ironie tragique, c'est parce qu'elles se concentrent à la fin de l'œuvre: la tour où était gardé le trésor explose. Au moment du triomphe final de l'allégorie, le spectateur comprend que le mystère échoue, car il triomphe. Lorsque l'allégorie étouffe la vie indigne du mystère qui l'entoure, elle se compromet et se détruit elle-même. Le fait que le mystère tue les gens qui se sont battus pour le préserver serait peut-être justifiable dans cette version « de la philosophie corallienne ${ }^{28}$ professée par la Gardien et l'Étrange, mais leur trésor n'est que la fonction des actes et des convictions de ces gens qui doivent cesser d'être pour préserver le trésor :

Le culte et votre dévouement pour les obscurs trésors

sont la plus haute valeur, le bijou le plus pur

du Trésor ${ }^{29}$

Ou :

le trésor exige de vous

que vous soyez inflexibles... Il vit dans les profondeurs

par la pureté de votre âme! Il s'y mire...

mais sans elle, comme un soleil pour les aveugles, le trésor mourra. ${ }^{30}$ 
Le trésor meurt avec les gens et la délivrance du trésor par rapport à ceux-ci signifie sa ruine ultime, parce qu'il vient d'eux et, juste avant la catastrophe, la démoniaque hypocrisie du style allégorique se révèle : «Agis!» - l'Étrange appelle le Gardien qui a décidé de faire exploser la tour avec le trésor et les gens, mais il hésite encore: «La promesse... J'ai dit que je partirais pendant la nuit... » L'Étrange réplique : «Encore un jour... » Le Garde résout le dilemme moral par une allégorie soignée :

Là-bas, la nuit éternelle est tombée

dans les âmes... Je jette l'effroi dans les ténèbres !

Que la menace du feu éternel fouette la nuit ! ${ }^{31}$

11 Staff n'explique pas si l'allégorie est mauvaise en tant que telle ou si c'est l'acte visant à intégrer la sagesse allégorique dans la vie qui est mal. Après l'intermède comique au style journalistique et satirique, les tirades finales des héros principaux sonnent déjà faux. Leur pathos se transforme en exaltation, snobant la virginité et l'idéalisme derrière lesquels se cache une méfiance fanatique à l'égard des gens. Il n'y a jamais eu de trésor, puisque (tous) les gens n'ont jamais été suffisamment purs et que leurs cœurs n'ont jamais produit d'image impeccable du trésor, s'il convenait de préparer une sortie de secours par avance 32 .

12 Ainsi, la pièce réflexive de Staff traite - comme Brzozowski le postule dans son compterendu et contrairement à ce qu'il lui reproche - de la relation tragique ou post-tragique de la vision de l'acte ${ }^{33}$; elle contient aussi une autocritique ou auto-compromission du style allégorique, bien que différente de la critique avancée par Brzozowski. Celui-ci fait tout pour ne pas percevoir cette autocritique de la pièce. S'il l'avait perçue, il serait devenu le spectateur de la compromission de ses propres idées. Il préfère toutefois répéter ses propres idées dans son style explosif et exubérant. Seulement, en refusant ainsi de se reconnaître dans la pièce, il ne fait qu'aggraver son cas, accentuant sa ressemblance avec les personnages de la pièce. En effet, Le Trésor de Staff ne contient pas seulement les conceptions de Brzozowski, mais aussi ses manières stylistiques. L'Étrange dit par exemple: "Mais ceux qui tomberont sous ce fardeau ressusciteront $! »^{34}$ Le Gardien : « Parce qu'ayant perdu le trésor, ils le possèderont. » ${ }^{35} \mathrm{Au}$ sujet d'Anton Čehov, Brzozowski affirme : « Il a oublié que seul celui qui sauve son âme la perd $»^{36}$ - se perdant dans l'action bien entendu. Dans la recension du Trésor, dans laquelle il exige le plus de vie possible, il oublie le prototype de ces paradoxes de prédication (Lc 9, 24): «Car quiconque veut préserver sa vie la perd. » Il tourne en allégorisme.

\section{L'allégorie et le fétiche du travail}

13 Brzozowski serait d'accord avec Irzykowski pour dire qu'il n'y a pas moyen de trouver ses propres conceptions dans une œuvre sans trouver en même temps son propre style. Leur querelle a été rendue possible par une conception commune de la langue - c'est en tout cas ce qui ressort de leurs déclarations - dont les deux facettes sont constituées de l' humboldtisme et de l'éthisme de la forme. L'humboldtisme ${ }^{37}$ se transforme d'ailleurs librement en éthisme de la forme dans "Niezrozumialcy" (Les incompréhensibles) d'Irzykowski : « Hebbel considère la parole comme la même catégorie de saisie du monde que l'espace et le temps, et affirme que chaque œuvre découle du contact d'éléments individuels inhérents à la nature avec des éléments généraux inhérents à la société. ${ }^{38} \mathrm{Il}$ existe un discours qui exclut effectivement l'acte et le condamne à l'immaturité, parce que justement une temporalité spécifique, dit Benjamin, caractérise l'allégorie en tant 
que forme d'expression qui exclut les moments décisifs ${ }^{39}$. Eu égard à l'humboldtisme commun aux deux critiques, Irzykowski peut parler globalement des effets «stylistiques et moralistes $»^{40}$ de Brzozowski et examiner ces effets stylistiques et éthiques, en s'attendant à ce que le « moyen d'exposer » suffise à concrétiser le programme de travail et de responsabilitét ${ }^{41}$. Et quand, déçu par le style de Brzozowski, Irzykowski critique «l'horrible manière » de ses «études à la limite entre la philosophie et la poésie »"2 , il le fait précisément à cause de l'infidélité de Brzozowski par rapport à son humboldtisme déclaré: les frontières de la langue de Brzozowski ne sont pas les frontières de sa connaissance. Quelque chose d'inexprimable, un " élément » tourbillonne en dehors de la conscience des gens. Brzozowski sait exactement ce que $c^{\prime}$ est $^{43}$ et il utilise tout un arsenal de styles élevés pour éveiller dans l'imagination du lecteur une image vacillante de ce quelque chose d'indicible à l'aide d'un langage " de l'exclu, du simplifié, du falsifié " ${ }^{44}$, si accessible à l'intuition de Brzozowski. Un humboldtisme conséquent, c'est-à-dire renonçant à l'intuition non discursive en faveur de l'agnosticisme et du « risque métaphysique ", doit permettre à Brzozowski de saisir que, comme le processus créatif est par essence un processus métaphorique ${ }^{45}$, les grandes métaphores, telles que Le Trésor de Staff, en démontrant leur propre pouvoir métaphorique, présentent un modèle de travail en tant que tel. Et Le Trésor fournit d'autant plus une critique du travail de Brzozowski dans les deux sens du terme : de son concept de «travail » et de sa profession d'intellectuel engagé.

À son tour, le deuxième principe commun des critiques - l'éthisme de la forme - est en fait basé sur l'hypothèse que la perfection de la forme résulte de l'éthos (du caractère) de l'auteur ${ }^{46}$. Cette hypothèse tacitement adoptée a donné l'expressionisme de Benedetto Croce, Leo Spitzer ou encore Karl Vossler qui a fait de la stylistique un domaine directeur des sciences historiques, donnant accès à l'expérience historique qui s'est trouvée une expression et qui peut grâce à cela être transmise au lecteur. Dans le cadre de ses recherches sur le style de Brzozowski, Irzykowski a découvert que l'éthisme changeait l'histoire en pièce de théâtre, puisqu'il n'ajoute rien aux considérations sur l'éthos de $L a$ Poétique d'Aristote ou à celles sur le caractère dramatique réussi de l'esthétique d'Hegel. Le caractère n'est qu'une fonction de l'action. "Dans le caractère et l'action de Brzozowski, il n'y a aucune pause $»^{47}$, tonnait Irzykowski devant le tribunal publique au moment de "l'affaire Brzozowski", alors qu'il présentait le penseur comme un héros tragique solitaire (le héros doit être solitaire, Brzozowski et lui-même sont tous les deux d'accord sur ce sujet). Pendant les périodes plus calmes, quand il ne fallait pas défendre Brzozowski face à l'opinion publique, Irzykowski ne voyait déjà plus en Brzozowski qu'un personnage allégorique comparable à l'Étrange et au Gardien, s'exprimant dans une langue tout aussi allégorique. Brzozowski ne voulait pas découvrir que le "trésor » (un accessoire absent) correspondait à son " point d'appui d'Archimède » ${ }^{48}$, c'est-à-dire au «travail »; que son style, forgé de manière à conserver le plus possible de parole vivante à l'écrit, se transformait en allégorie ; que lui-même devenait un spectateur passif et un metteur en scène autour duquel défilait une procession d'allégories.

Le trésor de la pièce de Staff est ce même hybride fait d'essentialisme et de fonctionnalisme, de trop-plein de définitions et de vide de sens, comme le «travail» de Brzozowski. Déjà en 1910, Wilhelm Feldman a pertinemment indiqué que le «travail » de Brzozowski était une étrange hypostase ${ }^{49}$, utilisant pour ce faire une construction rappelant les ultérieures ressemblances de famille de Ludwig Wittgenstein ${ }^{50}$. Nous appelons «travail» des activités très diverses - spirituelles et corporelles - qui se 
ressemblent à certains égards, et pas du tout à d'autres. Il n'y a pas de membre de la famille qui aurait un ensemble identique de traits.

Stanisław Wyspiański a eu besoin de douze jours pour écrire SĘdziowie (Les Juges), autant que le typographe pour préparer cette œuvre, autant qu'un porteur aurait eu besoin pour amener tous les exemplaires à un point donné. Chacun d'eux effectue un certain travail qui se reflète dans leur système musculaire et nerveux, mais chez chacun d'eux le travail est quelque chose d'autre ${ }^{51}$.

Extrait de ces activités hétérogènes, un travail un qui embrasse tout - la substance de l'être social - est un fantôme, une fiction, un concept vide. Son statut ontologique rappelle l'existence spectrale du trésor. Chez Brzozowski, le travail abstrait et vacillant est tragique par définition car il allie l'intention avec la performance physique, mais c'est l'allégorie qui devient la substance de ce tragique, comme chez Staff.

En revanche, eu égard à la relation au peuple, le trésor correspond structurellement à ce que Brzozowski appelle dans Legenda Młodej Polski (La Légende de la Jeune Pologne) un " mythe », un "tableau actif » qui déclenche et dirige l'activité, étant lui-même cette activité : «ce que l'on désire créer devient une force qui se crée par elle-même. " $^{52}$ Le mythe est finalement le travail sur le travail («la volonté de la volonté »), et la culture peut être appelée mythopoḯtique. Irzykowski s'inquiète de la réversibilité de la formule de Brzozowski. Le mythe est un travail, donc le travail s'avère un mythe. Cela mène à la fétichisation du travail qui acquiert les traits d'objet de culte. De plus, cette essentialisation mythique du travail résulte nécessairement de l'anti-essentialisme dans la version de Brzozowski. Staff démontre un processus analogue de fétichisation dans Le Trésor. La pièce boucle la boucle, en définissant le trésor en titre comme rien. Au début, le peuple doute qu'il se bat si héroïquement contre "l'inhumain " pour rien ${ }^{53}$. Au moment où le drame culmine, le Gardien crie au visage du Chef-traître ce qu'il adviendra quand le peuple atteindra le sous-sol du trésor :

Ils ne verront Rien! Et ils ne saisiront pas la lutte pour le trésor sacré, / Qui demeure une force tant qu'il n'est pas touché; / Qu'il ne fallait pas défendre! [Avant de définir ce qu'il est, NdlA] - Le Trésor des profondeurs / Disparaîtra s'il cesse de s'élever dans les hauteurs..$^{54}$

Dans l'introduction de son livre inachevé sur Wyspiański, Brzozowski parle de "l'entité extra-sociale » qu'il convient de retravailler, il explique qu'«elle peut être signifiée comme Dieu, la Nature, l'Inconnaissable, même comme Rien. Dans ce dernier cas, la connaissance, l'art, la morale sont des illusions, des rêves éveillés et la société colore, trouble ces rêves. $\aleph^{55}$ Le style paradoxal de Brzozowski qui combine anti-essentialisme et pathos prophétique, donnant en même temps un ardent agnosticisme, n'exclut pas de « rien » du discours, mais au contraire : il le bombarde d'un nombre infini de définitions jusqu'à ce que « rien » atteigne un excès de définitions, possible à ne maintenir que dans l'allégorie. Irzykowski lui reproche malicieusement que ses idées bifurquent, qu'elles " constitue une arme double tranchant ", et que même les nationaux-socialistes ont le droit voir en Brzozowski leur précurseur ${ }^{56}$.

Le Trésor de Staff semble toucher le paradoxe de la philosophie du travail de Brzozowski : le travail doit être infini et donc bouleverser d'une certaine façon tout ce qui limite la portée de sa définition. Cependant, il devient de ce fait une abstraction et, au lieu d'enraciner l'homme dans le monde et la vie, il le rejette dans le domaine de l'acte abstrait. 
D'après Irzykowski, la « dissimulation » est la forme de la pièce de Staff. La même chose peut être dite de la philosophie anti-essentialiste et essentialiste du «travail et de la liberté » de Brzozowski. La différence entre le drame et les écrits philosophiques se réduit au niveau de la réflexivité : l'action du drame allégorique de Staff représente un processus de fétichisation et la chute du fétiche, alors que Brzozowski reste un fidèle croyant du mythe qu'il s'est créé. Chez Staff, le trésor est ce que les gens rêvent qu'il soit : les gens attribuent à ce grand rien des propriétés intrinsèques, bien que le trésor soit en vérité une image fantasmatique de leur propre travail. Le trésor n'est pas seulement un produit de leur travail, le travail lui-même, substantialisé en mythe. Le travail qui devait falsifier les illusions du fétichisme de la marchandise, possible d'après Karl Marx grâce à la division et à la déformation du travail, paraît un dernier fétiche et - correspondant au trésor - une allégorie du style allégorique. Irzykowski compare le concept de «travail» de Brzozowski avec l'idole du Moloch qui contraint à la responsabilité totale ${ }^{57}$.

\section{Le socialisme dans le masque du démasquateur}

21 Michał Głowiński a décrit le style de Brzozowski comme une intrusion récurrente de phrases aphoristiques dans une parataxe potentiellement infinie fondée sur la répétition et l'ellipse ${ }^{58}$ ou - comme l'a résumé Irzykowski - de variations et de répétitions qui trahissent «la préoccupation de l'auteur» et «l'incompétence du savant ${ }^{59}$. Selon Głowiński, au niveau de la composition, la « règle du premier plan » correspondait à la parataxe et la précisait ; n'importe quoi devient objet du discours, revêt une importance primordiale et écarte tous les autres sujets de la scène. En effet, comme le voulait Hebbel, la langue correspond à l'espace-temps, dans ce cas-ci à l'espace-temps allégorique du Tauerspiel, dans lequel chacune des allégories participant au relais délivre de longs monologues pour disparaître. Le style allégorique se caractérise par une superposition sur le substantif qui est censé soumettre le syntagme. Irzykowski parle de la construction des expressions dans la phrase de Brzozowski: «Le fait de mettre des propositions relatives, souvent longues, devant le nom est typique de Brzozowski. En principe, c'est une construction assez étrangère à la langue polonaise. Grâce à elle, Brzozowski parvient toutefois à produire des effets stylistiques particuliers, quand un substantif donné finit par se distinguer dans toute sa force, réunissant d'une certaine manière en son sein tout le sens de la phrase. ${ }^{60} C^{\prime}$ est en revanche une construction naturelle pour la langue allemande, dans laquelle les substantifs s'écrivent avec des majuscules depuis l'époque baroque, l'âge d'or de l'allégorie. En outre, le style allégorique demande exclusivement des grands quantificateurs et des mots clés. Irzykowski énumère les expressions préférées de Brzozowski : « chaque », « tout », « jamais », « sans cesse ${ }^{61}$, «forger », « tragique $»^{62}$. Même Richard Avenarius était censé être tragique; l'heureuse vie à venir au nom de laquelle nous devrions travailler, était censée être tragique ${ }^{63}$.

Les observations stylistiques d'Irzykowski suggèrent que le caractère oratoire universellement reconnu du style de Brzozowski changeait son acte d'écriture en un monodrame allégorique. En donnant les constituants "rhétoriques» du style de Brzozowski - les parataxes, les énumérations, les exclamations, etc. -, Głowiński les répartit en différents "rôles »-styles ${ }^{64}$ (de l'enseignant, du pamphlétaire, de l'herméneute), préparés par ce «talent lyrico-épique pratiquant de la poésie didactique » ${ }^{65}$ - comme l'appelait Irzykowski -, se vantant « de tomber sans cesse dans l'excès ${ }^{66}$. Quand Irzykowski décrit "l'éloquence $»^{67}$ de Brzozowski, il lui retourne son propre 
argument de la recension de Pałuby (La Chabraque) : Brzozowski reprochait à Irzykowski que le «sens du rôle» ne fût possible que s'il n'y avait pas d'acte, puisque «l'absence d'absolutisation des idéaux » impliquait que tout idéal s'estompât dans une possibilité, une parmi d'autres ${ }^{68}$. Irzykowski prouve que Brzozowski faisait l'acteur sans avoir le " sens du rôle » au cours de ses " mises en scène de la vie de la pensée " ${ }^{69}$ ou, pire, avec un sens du rôle de conquérant de l'action: aucune de ses stratégies ne pouvait faire de son trésor - le travail - quelque chose d'un petit peu plus concret et vivant que l'allégorie. Le fait qu'une des nombreuses possibilités soit devenue la seule pendant une courte période ("la règle du premier plan») n'a pas empêché Brzozowski de faire l'acteur, bien au contraire : cela l'a projeté dans une variante très conventionnelle, le défilé d'allégories.

Face à la nation, Brzozowski n'a pas seulement incarné les rôles de pédagogue et de prédicateur, il a aussi mis en scène tout un spectacle d'incarnations de l'abstraction : « Il a réactivé les squelettes de l'abstraction et les a présentés au public comme de puissants éléments qui détruisent et commandent. ${ }^{70}$ Son attitude envers une audience qui lit ou qui écoute réalise le modèle du quatrième mur transparent de la scène : il était orienté vers l'auditeur, et non le lecteur, même quand il écrivait, mais il demeurait incapable de dialoguer avec le public ${ }^{71}$, et l'absence de sens du rôle et le fait d'écouter sa propre voix quand il «a fait parade $»^{72}$, l'ont précipité dans des péripéties tout droit sorties de «la tragédie du spectateur » qu'il décrivait à propos de Čehov. Un autre mur a poussé entre lui et la vie :

Ses principes de travail conviennent à tout ou à rien. Tendant vers les profondeurs du grand mouvement, il s'est trouvé tout à coup et involontairement au centre du cercle dans lequel tout est parfaitement immobile et confortable. Tout autour de lui, cependant tout tournait dans différents circuits et il s'est imaginé qu'il avait atteint l'axe du monde. ${ }^{73}$

Les personnages des romans de Stanisław Przybyszewski ne sont pas les seuls à être des allégories : « des caractères soudés, de la masse, créatrice de l'homme $»^{74}$ se fondant dans un Homme dont les protagonistes sont des aspects ${ }^{75}$. De plus, il observe la vie littéraire avec les yeux d'un metteur en scène-allégoriste : la reconnaissance de Brzozowski est « une reconnaissance dans les objectifs du système » et il transforme la littérature en un grand drame allégorique ${ }^{76}$. En conséquence de la division du travail qui se transforme imperceptiblement en une "répartition des rôles»" le statut de "signe», de " symptôme " ${ }^{78}$, «d'expression ", « d'incarnation » d'un certain aspect des forces occultes de l'histoire revient à chaque auteur dans le système de la littérature. Ainsi, le Staff si éthéré de La Légende de la Jeune Pologne joue «l'opposition et le complément » du «fils de la terre » de Jan Kasprowicz ${ }^{79}$. L'ensemble démasque le caractère abstrait et allégorique de toute l'époque de la Jeune Pologne qui a produit des féeries de pré-textes idéologiques pour fuir le travail et le « travail ».

Irzykowski écrit que "La Légende de la Jeune Pologne a été un des sommets de la pensée démasquatrice européenne qui a culminé chez Marx au XIX ${ }^{e}$ siècle et s'est achevée - s'estelle vraiment achevée ? - chez Freud au $x^{e}$ siècle ${ }^{80}$. L'action de démasquer ne peut pas s'achever, tout comme il n'est pas possible de retenir l'allégorèse qui traduit tout en loi connue par l'intuition ${ }^{81}$, une fois qu'elle est lancée : Brzozowski a lui-même été la victime de Bronowicz (Julian Brun), Andrzej Stawar, Stefan Kamieński ${ }^{82}$, plus précisément des penseurs socialistes qui ont fait de lui « une marionnette de l'histoire ». L'observation de cette allégorèse infinie a permis à Irzykowski de formuler une règlementation définissant la relation entre la littérature et le socialisme : la critique marxiste ou plus largement la 
critique socialiste est calquée sur le drame allégorique : le phénomène littéraire joue le rôle de "symbole ", " de phénomènes plus vastes et il est lié à eux comme la scène du drame à l'idée du drame, mais jamais sans reste $»^{83}$.

Le libéralisme n'ouvre pas de sortie de l'impasse de l'allégorie. Au contraire, le libéralisme classique a affirmé l'allégorie sous la forme d'une personne morale abstraite, alors que le socialisme scientifique a été créé pour répondre à la vie allégorique des sociétés après une révolution qui n'avait été que politique. Or, le jeune Marx qui voyait des «comédies », des "farces» tout autour de lui, une parodie de l'ancien régime $e^{84}$ dans l'Allemagne contemporaine et une parodie de la Rome républicaine dans la France révolutionnaire, et qui exigeait une tragédie authentique ${ }^{85}$, ne reconnut dans le citoyen ${ }^{86}$ de la société civile " qu'un homme artificiel abstrait », " une personne allégorique » ${ }^{87}$. Irzykowski est d'accord avec le diagnostic de Marx, suivant lequel le monde après une révolution politique est un monde d'allégories désincarnées, si bien qu'il affirme aussi que Marx ne pouvait manquer de transformer l'allégorie en concret tragique, puisqu'il a repris du libéralisme bourgeois exactement ce qui anéantit la personne dans un signe allégorique de l'humanité. Il a repris en l'occurrence le concept de nécessité, équivalent de "l'automatisme ${ }^{88}$. Ayant appris d'Adam Smith et des autres classiques britanniques de l'économie à faire confiance à « l'automatisme » des sociétés ${ }^{89}$, le socialisme scientifique s'est réduit à l'historicisation de l'économie de sorte que le destin fût incarné dans l'histoire des moyens de production, poussant en direction d'une explication définitive de l'être social. Explication qui démasque toutes les solutions partielles comme nécessaires, et donc les mensonges compréhensibles qui ont joué un rôle déterminé dans le théâtre de l'histoire, poussant l'action vers une solution connue d'avance. En vérité, le libéral garde un avantage sur le socialiste dans la mesure où il conserve la "conscience du rôle » et qu'il sait même que c'est un rôle allégorique. Mais il est peu probable que le libéralisme soit capable de se retenir de faire la morale ${ }^{90}$, morale qui métamorphose le libéralisme en impérialisme, aspirant à la fin de l'histoire par le triomphe définitif de la vérité sur la nature de l'homme-producteur. Et tout chantage moral - pas seulement le chantage de Brzozowski par la responsabilité totale - est ressenti par Irzykowski comme un « attentat contre les travaux directs de la pensée $»^{91}$.

En tant que critique, Irzykowski ne voit pas de sortie de l'impasse allégorique. Son besoin de mesurer le fond de l'œuvre ne fait qu'arracher aux démasquateurs leurs masques, derrière lesquels se cachent les allégoristes et les metteurs en scène du Tauerspiel, usurpant la connaissance de la nécessité. Seulement, pour avoir soupçonné les soupçonneux, Irzykowski paye en rejoignant leur cadavre. D'où la sympathie d'Irzykowski pour Staff, « l'allusionisme » littéraire, le " parabolisme », ainsi que pour Brzozowski dont il parlait de la manière la plus intéressante quand il se servait de l'allusion et de l'insinuation.

La tragédie de Staff Le Trésor a servi à Brzozowski et à Irzykowski de prétexte pour formuler une théorie du discours capable de passer de l'ordre des mots aux actes et aux choses. Les enjeux étaient de taille : la décadence de la fin du XIX ${ }^{e}$ siècle et du début du Xx siècle a été définie, tant dans la dimension culturelle générale que strictement politique, comme l'incapacité à passer de la parole à l'acte. En revanche, on a supposé qu'un style de parler adéquat serait un symptôme témoignant de l'ancrage du discours dans la vie et le vécu : seul un tel discours, un discours sorti de la vie, aurait une chance d'accomplir de réels changements dans la vie. La théorie de la tragédie devait en principe examiner les possibilités de passer de la théorie aux actes. Quand Brzozowski décrivait le style non 
tragique de Staff et qu'Irzykowski comparait le style de Staff au style de Brzozowski, tous les deux s'amusaient à imputer à leur adversaire le caractère non pratique du discours, le détachement de la parole par rapport à la vie. Le jeu a finalement conduit à ce que l'accusateur soit lui-même accusé, privé de la possibilité d'influer par le verbe sur le cours des événements. Les programmes politiques et économiques les plus pratiques s'avèrent un bavardage vain, livré à la face des événements qui suivent leur cours. Le présent article avait pour objet de décrire ce style qui tend vers cette dimension pratique impossible ainsi que les jeux d'accusations. Nous avons vu le retournement ironique des paroles pathétiques de Brzozowski que nous avons mises en exergue en guise de leitmotiv pour l'ensemble de l'article : les auteurs, «prophètes ", «bardes ", rêvant de l'action par les mots, enlevés par le style, ont cessé d'être des auteurs et sont devenus des « héros et des victimes ", mais non pas de la tragédie, juste du Tauerspiel allégorique. C'est ce destin qu'a présenté Staff dans sa « tragédie ».

\section{NOTES}

1. Brzozowski St., "Miriam - zagadnienie kultury» (Miriam : la question de la culture), in : Programy i dyskusje literackie okresu Młodej Polski (Programmes et discussions littéraires de l'époque de la Jeune Pologne), sous la direction de Podraza-Kwiatkowska M., Ossolineum, Wrocław, 1973, p. 613.

2. Staff L., Skarb. Tragedja $w$ trzech aktach (Le Trésor. Tragédie en trois actes), Towarzystwo Wydawnicze J. Mortkowicz, Varsovie, 1932.

3. Brzozowski St., Kultura i życie. Zagadnienia sztuki i twórczości w walce o światopogląd (La Culture et la vie. Les questions de l'art et de l'œuvre dans la lutte pour la conception du monde), Państwowy Instytut Wydawniczy, Varsovie, 1973, pp. 146 \& 702. Cf. Królica A., Postawa wychowawcy a postawa klerka: Stanisława Brzozowskiego i Karola Irzykowskiego spór o światopoglądowe podstawy krytyki literackiej (L'attitude de l'éducateur et l'attitude du clerc :la querelle de Stanisław Brzozowski et de Karol Irzykowski au sujet des fondements philosophiques de la critique littéraire), Wydawniczy Uniwersytet Opolskiego, Opole, 2000, p. 28.

4. Hegel G. W. Fr., Werke (Travaux), Suhrkamp, Francfort-sur-le-Main, 1979, pp. 342-354 \& 512-544.

5. J'en parle plus longuement dans ma thèse d'habilitation, actuellement en préparation, ayant pour titre: «Tragödie und Revolution. Die kritischen Theorien der Tragödie als Praxeologie in Deutschland und in Polen 1789-1848 » (La Tragédie et la révolution. Les théories critiques de la tragédie comme praxéologie en Allemagne et en Pologne 1789-1848).

6. J'ai fait le choix de ne pas distinguer « l'acte » et le «travail ». D'un côté, je suis d'accord avec une des principales thèses de l'excellente monographie d'Andrzej Walicki (Walicki A., Stanisław Brzozowski - droga myśli [Stanisław Brzozowski : le cheminement de sa pensée], Universitas, Cracovie, 2011), qui décrit le « cheminement de la pensée » de Stanisław Brzozowski comme un passage d'un "acte » abstrait au travail, ce dernier étant toujours un travail sur un matériel donné dans un environnement défini. Toutefois, comme nous le verrons, le style de Brzozowski la manière dont il développe les figures de la pensée et des mots - sabote définitivement la distinction entre le travail et l'acte.

7. Brzozowski St., Kultura i życie, op. cit, p. 203. 
8. Irzykowski K., Czyni słowo oraz Fryderyk Hebbel jako poeta konieczności. Lemiesz i szpada przed sĄdem publicznym. Prolegomena do charakterologii (L'acte et la parole ainsi que Fryderyk Hebbel en tant que poète de la nécessité. La lame et l'épée en jugement public. Prolégomènes pour une caractérisation), Wydawnictwo Literackie, Cracovie, 1980, pp. 376, 490 \& 609. Voir également Walicki A., op. cit., pp. 44-50.

9. Brzozowski St., Legenda Młodej Polski. Studya o strukturze duszy kulturalnej (La Légende de la Jeune Pologne. Étude sur la structure d'une âme culturelle), Księgarnia Polska Bernarda Połonieckiego, Cracovie-Wrocław, 1983, pp. 452-453.

10. Brzozowski St., Kultura i zycie, op. cit., p. 205.

11. Ibid., p. 206.

12. Ibid., p. 207.

13. Irzykowski K., "Motywy dramaturgii Staffa " (Les Motifs de la dramaturgie de Staff), in : Pisma teatralne (Écrits théâtraux), t. II, Wydawniczy Literackie, Cracovie, 1995, p. 617.

14. Ibid., p. 618.

15. Ibid., t. I, pp. $276-279$.

16. Brzozowski St., Pamį̨tnik (Mémoires), Wydawniczy Literackie, Cracovie-Wrocław, 1985, p. 149.

17. Benjamin W., Gesammelte Schriften (๕uvres complètes), t. I, Tiedemann, Suhrkamp, Francfortsur-le-Main, 1976, p. 1238 ; Bathi T., "Theories of Knowledge: Fate and Forgetting in the Early Works of Walter Benjamin » (Théories de la connaissances : Le destin et l'oubli dans les premières œuvres de Walter Benjamin), in : Benjamin's Grounds. New Readings of Walter Benjamin (Le territoire de Benjamin. Nouvelles lectures de Walter Benjamin), sous la direction de Nägele R., Wayne State University Press, Detroit, 1986, p. 80.

18. Ibid., pp. 238-276 \& 337-340.

19. Symbol. Grundlagetexte aus Ästhetik, Poetik und Kulturwissenschaft (Le Symbole. Textes fondamentaux d'esthétique, de poétique et d'études culturelles), sous la direction de Berndt Fr. \& Drügh H. J., Surkamp, Francfort, 2009, pp. 223-291.

20. Staff L., op cit., p. 5.

21. Brzozowski St., « Monistyczne pojmowanie dziejów i filozofia krytyczna » (La compréhension moniste de l'histoire et la philosophie critique), in: Kultura i życie, op.cit., pp.575-576; Irzykowski K., Słońwśród porcelany. Lżejszy kaliber (Un éléphant entouré de porcelaine. Un calibre plus léger), Wydawniczy Literackie, Cracovie, 1976, p. 321.

22. Staff L., op. cit., p. 22.

23. Irzykowski K., Walka o treść. Beniaminek (L'Aspiration à l'essence. Benjamine), Wydawniczy Literackie, Cracovie, 1976, pp. 151-152.

24. Christiansen Br., Philosphie der Kunst (La Philosophie de l'art), Clauss und Feddersen, Hanau,1909, pp. 243 et suiv. ; Wóycicki K., Jedność stylowa utworu poetyckiego (L'unité stylistique de l'œuvre poétique), Drukarnia K. Kowalewskiego, Varsovie, 1915, pp. 11 et suiv.

25. Aust H., Haida P. \& Hein J., Volksstück. Vom Hanswurstspiel zum sozialen Drama der Gegenwart (Jeu populaire. Du jeu de bouffon au drame social actuel), Beck, Munich, 1996.

26. Staff L., op. cit., p. 19.

27. Ibid., p. 20.

28. Irzykowski K., Stońwśród porcelany. op. cit., p. 506.

29. Staff L., op. cit., p. 93.

30. Ibid., p. 87.

31. Ibid., p. 158.

32. Les explosifs réunis dans la tour témoignent de la non-existence du trésor, car le Garde a le trait laid et impur de la suspicion.

33. Brzozowski St., Kultura i zycie. op. cit., p. 205.

34. Staff L., op. cit., p. 45. 
35. Ibid., p. 81.

36. Brzozowski St., Kultura i życie, op. cit., p. 269.

37. Nycz R., JĘzyk modernizmu. Prolegomena historycznoliterackie (La langue du modernisme. Prolégomène hostorico-littéraire), Fundacja na Rzecz Polski, Wrocław, 2002, pp. 129 et suiv.

38. Irzykowski K., Czyni słowo, op. cit, p. 482.

39. Benjamin W., Gesammelte Schriften, op.cit., p.340. L'allégorie peut être sauvée justement quand elle reconnaît son propre mal et sa propre impuissance, le vide du savoir qu'elle porte. Tel est justement le final du Trésor et d'Ursprung des deutschen Trauerspiels (L'Origine de la tragédie allemande) de Walter Benjamin.

40. Irzykowski K., Czyni słowo, op. cit, p. 273.

41. Irzykowski K., Słońwśród porcelany, op. cit., p. 269.

42. Irzykowski K., Czyni słowo, op. cit, p. 501.

43. Ibid., pp. 297-298.

44. Brzozowski St., PamiĘtnik, op. cit., p. 148.

45. Irzykowski K., Walka o treść, op. cit., pp. 66-69.

46. Ibid., p. 142 ; Irzykowski K., Czyni słowo, op. cit, pp. 402-403.

47. Ibid., p. 183.

48. Brzozowski St., Idee. WstĘp do filozofii dojrzałości dziejowej, Wydawnictwo Literackie, Cracovie, 1990, p. 172.

49. Feldman W., «Przeciw filozofii pracy» (Contre la philosophie du travail), in : Programy $i$ dyskusje w okresie Młodej Polski, op. cit., pp. 654-656.

50. Wittgenstein L., Philosophische Untersuchungen (Investigations philosophiques), Wissenschaftliche Buchgesellschaft, Francfort, 2001, §§ 65-71.

51. Feldman W., art. cit., p. 655.

52. Brzozowski St., Legenda Młodej Polski, op. cit., p. 253.

53. Staff L., op. cit., pp. 17 \& 19 .

54. Ibid., p. 154.

55. Brzozowski St., Stan. Wyspiański (Wydanie pośmiertne) (Stan. Wyspiański [Édition posthume]), sous la direction de Irzykowski K., M. Haskler, Stanisławów, 1911, p. 9.

56. Irzykowski K., Pisma rozproszone (Æuvres dispersées), t. III, Wydawnictwo Literackie, 1999, p. 517.

57. Irzykowski K., Czyni słowo, op. cit, pp. 493, 180 \& 301.

58. Głowiński M., «Wielka parataksa. O budowie dyskursu w Legendzie Młodej Polski Stanisława Brzozowskiego » (La grande parataxe. La construction du discours dans La Légende de la Jeune Pologne de Stanisław Brzozowski), in : PamiĘtnik Literacki,nº 4/1991, pp. 54-70. Cf. Markiewicz H., «Strategie krytycznoliterackie» (Les stratégies de la critique littéraire), in: Markiewicz H., Czytanie Irzykowskiego (La lecture d'Irzykowski), Universitas, Cracovie, 2011, passim.

59. Irzykowski K., Czyni słowo, op. cit, p. 375.

60. Irzykowski K., Pisma rozproszone, op. cit., t. III, p. 191.

61. Irzykowski K., Czyni słowo, op. cit, p. 375.

62. Irzykowski K., Pisma rozproszone, op. cit., t. IV, p. 384.

63. Irzykowski K., « Dostojny bzik tragiczności» (La digne vogue du tragique), in : Czyni słowo, op. cit, pp. 246-257. Cf. Wyka M., «O niektórych terminach krytycznych Legendy Młodej Polski », in : Wyka M., Czytanie Brzozowskiego (Lire Brzozowski), Universitas, Cracovie, 2012, passim.

64. Głowiński M., art. cit., p. 60.

65. Irzykowski K., Czyni słowo, op. cit, pp. 498, 488, 496 \& 501.

66. Ibid., pp. 301-302.

67. Ibid., p. 485.

68. Brzozowski St., Eseje i studia o literaturze (Essais et études sur la littérature), t. 1, Zakład Narodowy im. Ossolińskich, Wrocław, 1990, p. 152. 
69. Irzykowski K., Pisma rozproszone, op. cit., t. IV, p. 368.

70. Irzykowski K., Czyni słowo, op. cit, p. 478.

71. Irzykowski K., Pisma rozproszone, op. cit., t. IV, p. 389.

72. Irzykowski K., Czyni słowo, op. cit, p. 603.

73. Ibid., p. 496.

74. Irzykowski K., Słońwśród porcelany, op. cit., p. 191.

75. Irzykowski K., Czyni słowo, op. cit, pp. 598-600.

76. Ibid., p. 344.

77. Ibid., p. 490.

78. Ibid., p. 497.

79. Brzozowski St., Legenda Młodej Polski, op. cit., p. 450.

80. Irzykowski K., Pisma rozproszone, op. cit., t. IV, p. 208.

81. Irzykowski K., Stońwśród porcelany, op. cit., p. 308.

82. Ibid., p. 296.

83. Ibid., p. 304.

84. En français dans le texte.

85. Marx K., «Zur Kritik der Hegelschen Rechtsphilosophie. Einleitung " (Introduction à la critique hégelienne de la philosophie du droit), in: Engels Fr. \& Marx K, Werke (Travaux), t. 1, Berlin 1976, pp. 378-391; Marx K., «Der achtzehnte Brumaire des Louis Bonaparte " (Le 18Brumaire de Louis Bonaparte), in : Engels Fr. \& Marx K, op. cit., t. 8, pp. 115-123.

86. En français dans le texte.

87. Marx K., «Zur Judenfrage » (la question juive), in : Marx K., Engels Fr., Werke, t. 1, op. cit., p. 370.

88. Il serait d'ailleurs facile de montrer que le discours de Marx doit sa forme à la stylistique allégorique.

89. Irzykowski K., Pisma rozproszone, op. cit., t. IV, p. 133.

90. The Theory of Moral Sentiments (Théorie des sentiments moraux, 1759)d'Adam Smith prépare et consolide An Inquiry into Nature and Causes of the Wealth of Nations (Recherches sur la nature et les causes de la richesse des nations, 1776).

91. Irzykowski K., Słońwśród porcelany, op. cit., p. 297.

\section{AUTEURS}

\section{MICHAL MRUGALSK}

Eberhard Karls Universität Tübingen, DAAD-Gastprofessor 